\title{
Des détecteurs de traces à la radiobiologie : la contribution du LMN-AC
}

\author{
M. FROMM ${ }^{1}$, J.E. GROETZ ${ }^{1}$
}

(Manuscrit reçu le 6 novembre 2006, accepté le 28 février 2007)

\begin{tabular}{ll} 
RÉSUMÉ & $\begin{array}{l}\text { Nous présentons à travers l'historique du Laboratoire de Microanalyses Nucléaires } \\
\text { Alain Chambaudet (LMN-AC) les différentes thématiques de recherche entrant } \\
\text { dans le cadre de la dosimétrie des rayonnements ionisants et de la radiophysique. }\end{array}$ \\
ABSTRACT & $\begin{array}{l}\text { From solid state nuclear track detectors to radiobiology: a contribution from } \\
\text { LMN-AC. } \\
\text { Through the story of the Alain Chambaudet Nuclear Microanalysis Laboratory, the } \\
\text { research topics are outlined with relation to dosimetry and spectrometry of ionizing } \\
\text { radiations and radiobiology. }\end{array}$ \\
Keywords: & CR-39 / bystander effect / neutron spectrometry / DNA deposit \\
\hline
\end{tabular}

\section{Historique du laboratoire et des thématiques de recherche}

Deux ans après son recrutement comme Professeur, Alain Chambaudet crée en 1983 à l'université de Franche-Comté le Laboratoire de Microanalyses Nucléaires (LMN). La thématique fondatrice du laboratoire est basée sur l'utilisation des Détecteurs Solides de Traces Nucléaires (DSTN) à des fins de dosimétrie, de cartographie des particules chargées lourdes et de datation. Les premiers sujets abordés ont concerné l'analyse de traces de fission spontanée et induite après gravure chimique dans des cristaux d'hydroxy- et de fluoro-apatite en vue de déterminer leur âge géologique de formation et de comprendre le phénomène d'effacement partiel des traces latentes dites fossiles.

Le laboratoire s'équipe en spectromètres de rayonnements nucléaires et en dispositifs d'observation et d'analyse à l'échelle microscopique et utilise régulièrement la microsonde électronique. Il développe alors des collaborations, à la fois avec des industriels et des grands organismes de recherche, notamment le CEA et la DGA. Il est reconnu équipe d'accueil de Microanalyse des Matériaux (EA473) par le ministère de la Recherche. À coté des traces nucléaires dans les minéraux, celles gravées dans les polymères constituent un mode alternatif de

${ }^{1}$ Laboratoire de Microanalyses Nucléaires Alain Chambaudet, UMR CEA E4, université de Franche-Comté, 16 route de Gray, 25030 Besançon Cedex, France. 
dosimétrie des rayonnements alpha et des neutrons qui sera développé des années 1980 jusqu'à nos jours, incluant les moyens de simulation numérique, notamment pour la dosimétrie des neutrons.

En 1988, le LMN accueille à Besançon la $\mathrm{VI}^{\mathrm{e}}$ Conférence internationale sur la datation par traces de fission. À la fin des années 1980, avec un effectif plus important en permanents et doctorants, se développent des thématiques fortes comme l'étude du vieillissement des matériaux composites, l'étude de la porosité des matériaux géologiques et la réactivité en surface de capteurs de gaz, pour lesquelles le LMN est toujours reconnu. En 1993, le LMN accueille la deuxième conférence internationale sur la géochimie des gaz. S'associant au Laboratoire de Spectrométrie Hertzienne du Professeur Jean-Gérard Théobald, l'équipe de Microanalyse des Matériaux est forte de 16 permanents et de 17 doctorants en 1998, elle organise à Besançon la XIX ${ }^{\mathrm{e}}$ conférence internationale sur les Traces Nucléaires dans les Solides. En 1999, elle accueille les XVI ${ }^{\mathrm{es}}$ journées des LARD.

En septembre 2000, le CEA DAM de Valduc signe une convention avec l'Université de Franche-Comté, transformant l'équipe d'accueil en Laboratoire de Recherche Correspondant (LRC M07) du CEA avec la possibilité de transformation en UMR. Cette transformation sera effectivement signée le 15 décembre 2003 après évaluation par son conseil scientifique. Alain Chambaudet nous avait quitté en août 2002, laissant derrière lui une équipe qu'il avait formée et qui structure alors dans un premier temps la recherche autour des deux axes : «surfaces, interfaces et matériaux » et « interactions rayonnements nucléaires - matière ». En accueillant en 2003 deux nouveaux permanents, l'UMR CEA E4, Laboratoire de Microanalyses Nucléaires Alain Chambaudet, dirigée par Michel Fromm (université de Franche-Comté) et Catherine Treimany (CEA-Va), dotée de son comité de direction, évolue vers une nouvelle organisation en s'orientant suivant les axes :

- interactions rayonnements ionisants - matière ;

- capteurs de gaz;

- mécanismes moléculaires d'intérêt biologique.

L'effectif actuel est de trois professeurs, quatre maîtres de conférences, un ingénieur de recherche et un d'étude universitaires, un ingénieur CEA et trois chercheurs-ingénieurs CEA-Va rattachés à l'UMR, encadrant une dizaine de doctorants.

Sur le plan de l'enseignement universitaire, le LMN-AC est un laboratoire de chimie-physique, fortement impliqué dans les cours d'atomistique, de chimies générale, analytique, sous rayonnements, quantique, de photochimie et de spectroscopies. 
Depuis plus de vingt ans, partant des détecteurs de traces pour s'orienter vers la radiolyse et la radiobiologie, le LMN-AC a développé toute une série d'études en relation avec la radiophysique et la dosimétrie, et cet article a pour objectif d'en présenter les principales.

\section{Les détecteurs solides de traces nucléaires et leurs applications}

\subsection{Gravure chimique des traces nucléaires}

Le phénomène de gravure chimique préférentielle d'une trace d'ion lourd dans un solide a été mis en évidence en 1958 lorsque Young, utilisant comme détecteur le fluorure de lithium, publie sa découverte dans la revue Nature (Young, 1958). En 1975, Fleischer, Price et Walker ont rassemblé dans un important ouvrage leurs approches théoriques, les principes expérimentaux et des applications des traces nucléaires dans les solides à différents domaines des sciences (Fleischer et al., 1975). Dans la même période, deux groupes américains suggèrent d'utiliser un polymère à base de carbonate de diéthylène glycol, connu sous la dénomination commerciale de CR-39 (Cartwright et al, 1978 ; Cassou and Benton, 1978), pour la détection des traces d'ions rapides. Ce polymère transparent utilisé notamment dans l'industrie de la lunetterie offre à la fois une large gamme d'énergie $(\beta=\mathrm{v} / \mathrm{c})$ et de type d'ion (Z) détectable lorsqu'il est utilisé en tant que DSTN. De plus, les traces gravées dans le CR-39 sont très bien résolues sous un microscope optique en raison d'un fort contraste entre la partie gravée (sombre) et la partie indemne du polymère (claire). La figure 1 récapitule les différents domaines scientifiques dans lesquels le CR-39 a été utilisé jusqu'à nos jours.

La gravure chimique des traces nucléaires est une étape nécessaire si l'on désire observer, dénombrer et analyser la morphologie des traces produites par des particules lourdes chargées dans un solide à l'aide d'un microscope optique. L'étape de gravure consiste en une attaque préférentielle du matériau détecteur le long de la trajectoire de la particule, là où les modifications chimiques engendrées par le ralentissement de l'ion incident présentent avec la solution de gravure une réactivité spécifique. Pour la gravure chimique du CR-39, on utilise une base concentrée et chaude, généralement de l'hydroxyde de sodium ou de potassium à températures comprises typiquement entre 60 et $80^{\circ} \mathrm{C}$ et pendant plusieurs heures.

La zone sensible qui fait l'objet de la gravure préférentielle est appelée trace latente. Dans un matériau diélectrique isotrope tel qu'un polymère amorphe, la zone de dégâts latents est une région du détecteur, en première approximation de révolution axiale autour de la trajectoire de l'ion, dont la densité de dégâts est modulée suivant ses dimensions longitudinales et radiales par les processus de 


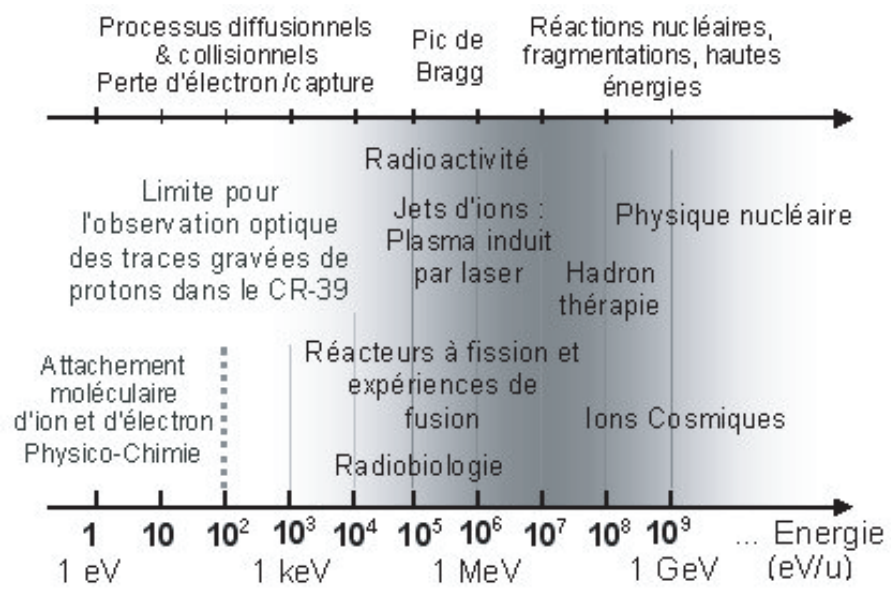

Figure 1 - Échelle d'énergie des ions et principaux domaines de la recherche scientifique où le DSTN CR-39 est utilisé.

Energy scale of ions and main research topics where the SSNTD CR-39 is used.

dépôt d'énergie, de relaxation, puis éventuellement par des variations ultérieures de l'environnement chimique au voisinage de la trace (gaz dissous, espèces volatiles, variation de l'atmosphère, etc.) ou encore de la température.

Quant à la phase de gravure chimique qui est réalisée ultérieurement, une description fidèle de son mécanisme est requise dès lors que l'on souhaite réaliser des expériences à caractère métrologique utilisant les DSTN (cartographie, dosimétrie, etc.). La gravure chimique est généralement décrite via deux vitesses spécifiques, celle d'attaque globale du matériau, $\mathrm{V}_{\mathrm{B}}$ (bulk), et celle spécifique à la trace, $\mathrm{V}_{\mathrm{T}}$.

La vitesse $V_{B}$ est mesurable de différentes manières, par exemple en déterminant simplement la perte de masse d'un échantillon non exposé aux radiations en fonction du temps d'immersion dans la solution de gravure (Fromm et al., 1993). Toutes les mesures réalisées indiquent une croissance exponentielle de cette vitesse avec la température et la concentration. Pour une température et une concentration données, $V_{B}$ est généralement supposée constante, notamment pour des durées de gravure de l'ordre de quelques heures.

La vitesse $\mathrm{V}_{\mathrm{T}}$ est mesurable et présente des variations (Ditlov et al., 2005), elle est cependant parfois considérée constante dans les applications usuelles des DSTN. Sa mesure a été réalisée avec des protons de différentes énergies dans le 


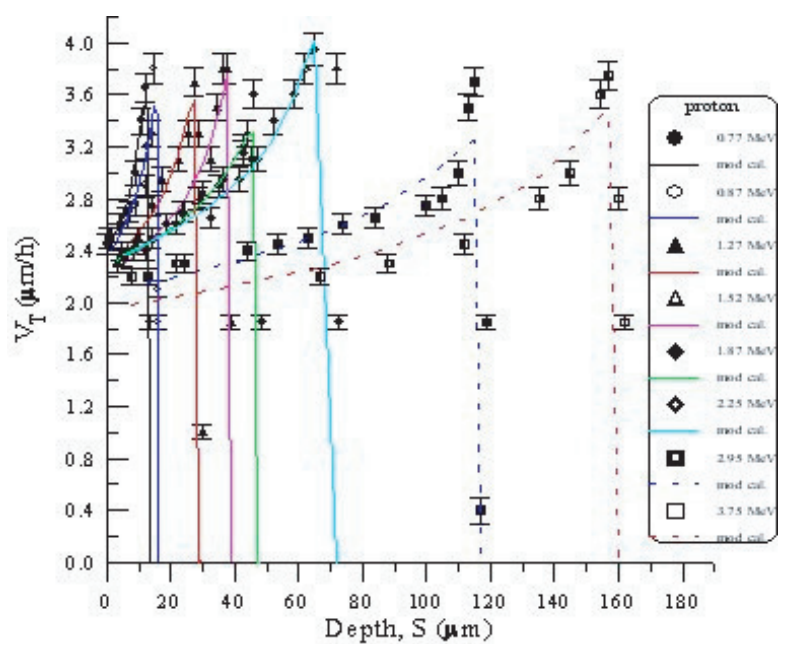

Figure 2 - Vitesses spécifiques de gravure $\left(V_{T}\right)$ mesurées dans le CR-39 pour des traces de protons d'énergie cinétique variant entre 0,77 et 3,75 MeV en fonction de la profondeur (S) dans le CR39. Les courbes représentent un calcul des vitesses spécifiques basé sur la théorie balistique avec comme variable physique le transfert restreint d'énergie $(R E L)_{\omega}$. La minimisation de l'erreur statistique a conduit à utiliser une coupure à $\omega=50 \mathrm{eV}$ pour optimiser le calcul de $V_{T}$ (d'après Ditlov et al., 2005).

Etching velocities $\left(V_{T}\right)$ measured in CR-39 for tracks from protons with a energy between 0.77 and $3.75 \mathrm{MeV}$ according to the depth in CR-39. Curves show a calculation of specific velocities based on the ballistic theory with the REL as variable. The minimization of the statistical deviation leads to a threshold frequency $\omega=50 \mathrm{eV}$ in order to optimize the $V_{T}$ calculation (from Ditlov et al., 2005).

CR-39. La figure 2 montre l'évolution de ces vitesses mesurées et recalculées en fonction de la profondeur.

Nous avons développé un modèle permettant de reproduire, le plus fidèlement possible, à l'échelle du micromètre, la formation des traces gravées. Ce modèle suppose une variation longitudinale de $\mathrm{V}_{\mathrm{T}}$ et une attaque globale constante et isotrope $\mathrm{V}_{\mathrm{B}}$ du CR-39 hors de la trace. On fait une analogie avec les ondes de Mach pour décrire l'action de la gravure chimique. Chaque point $\lambda$ de la trajectoire initiale de l'ion dans le polymère est la source d'une sphère de révélation dont le rayon est proportionnel au temps résiduel $\tau=\mathrm{t}-\mathrm{t}_{\lambda}$; $\mathrm{t}$ est le temps total de gravure, $\mathrm{t}_{\lambda}$ le temps nécessaire pour que la solution de gravure atteigne, à la vitesse $\mathrm{V}_{\mathrm{T}}$, un point $\lambda$ de la trace. Dans ces conditions, pour graver une trace, des sphères successives grandissent à vitesse constante et isotrope $\left(\mathrm{V}_{\mathrm{B}}\right)$.

Ce modèle rend bien compte de la variation de géométrie des traces gravées et de leur disparition au-delà d'un angle d'incidence critique du projectile. 


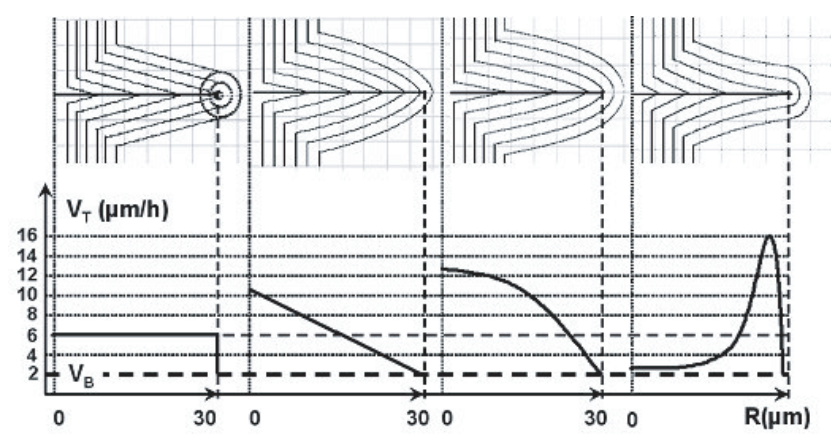

Figure 3 - Simulations du développement séquentiel de traces à l'aide de quatre fonctions de réponse $V_{T}$ pour un parcours $R=30 \mu m$. Une première vitesse constante égale à $6 \mu m . h^{-1}$. Une seconde décroissante linéaire ayant une valeur moyenne de $6 \mu m . h^{-1}$. Une troisième concave dont la valeur moyenne est supérieure à $6 \mu \mathrm{m} . \mathrm{h}^{-1}$. Une quatrième présentant un maximum et dont la valeur moyenne est $6 \mu \mathrm{m} . h^{-1}$. La vitesse $V_{B}$ est toujours fixée à $2 \mu m . h^{-1}$ et illustre typiquement celle mesurée avec le CR-39 plongé dans une solution de gravure composée d'hydroxyde de sodium $7,0 \mathrm{M}$ à $70^{\circ} \mathrm{C}$. Une vitesse moyenne de $6 \mu \mathrm{m} . h^{-1}$ est représentative d'un ion avec $Z \sim 2$, 3 pour une énergie cinétique de l'ordre de $5 \mathrm{MeV}$ (d'après Fromm, 2005).

Simulation of the sequential etching of a track with a range $R=30 \mu \mathrm{m}$ for $6 \mathrm{~h}($ step $=1 \mathrm{~h})$. From left to right, the used $V_{T}$ 's are a constant value of $6 \mu \mathrm{m} / \mathrm{h}$ (conical in shape model), a decreasing linear value (from $V_{T} \sim 10$ to $V_{B}=1.95 \mu \mathrm{m} / \mathrm{h}$ ) with an average value of $6 \mu \mathrm{m} / \mathrm{h}$, a decreasing power-like function (with an average $V_{T} \sim 9 \mu \mathrm{m} / \mathrm{h}$ ) and a Bragg-type function (average value of $6 \mu \mathrm{m} / \mathrm{h})($ Fromm, 2005).

L'enveloppe des sphères à un temps t correspond à la forme tridimensionnelle de la trace gravée. Un exemple d'application de ce modèle est présenté en figure 3, pour un angle d'incidence normal à la surface du DSTN. Dans cette figure, quatre différentes formes de vitesses de gravure sont choisies pour générer les coupes sagittales des traces gravées.

Différentes méthodes ont permis d'obtenir des images microscopiques bi- et tri-dimensionnelles de traces nucléaires gravées (Fromm et al., 1991). La méthode la plus récente consiste à imprégner le polymère après la gravure des traces avec un colorant fluorescent. De cette manière une image tri-dimensionnelle est obtenue sous éclairement laser par microscopie confocale (Meesen and Poffijn, 2001). Un accord tout à fait satisfaisant entre modèle et expérience a été obtenu.

Concernant les ouvertures de traces gravées dans le plan de la surface du détecteur, la figure 5 permet de comprendre l'évolution de leur géométrie en fonction de l'angle d'incidence de la particule. Nous avons établi les relations permettant de réaliser le calcul des paramètres géométriques d'une trace gravée en fonction du temps de gravure. Dès lors que l'on dispose d'une fonction 

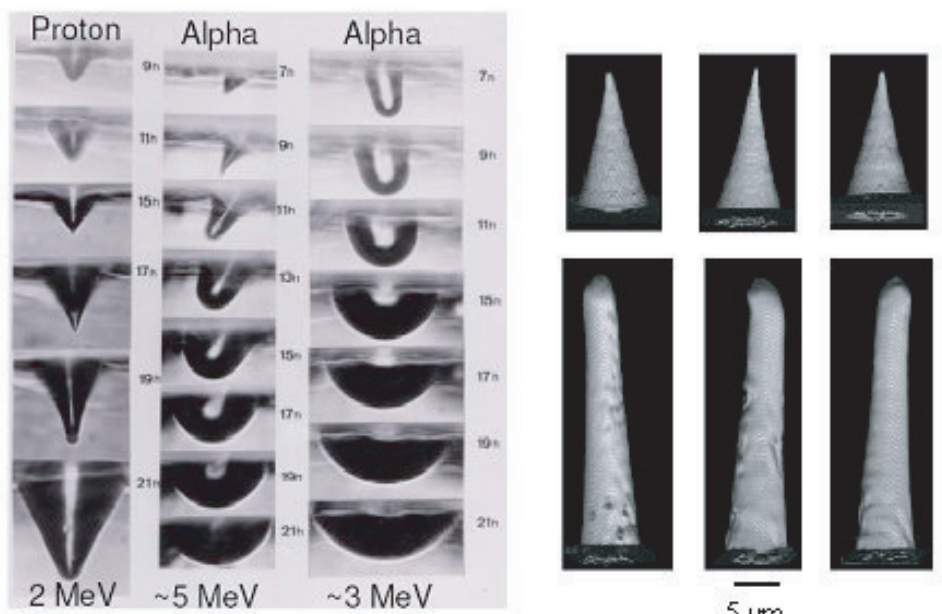

Lithium $10,8 \mathrm{MeV}$
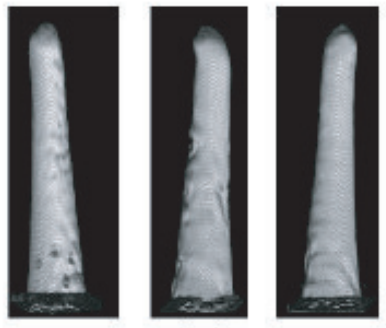

Carbone $22,5 \mathrm{MeV}$

Figure 4 - Profils obtenus par transmission optique et vues tridimensionnelles de traces gravées issues de particules ralenties dans le polycarbonate de diéthylène glycol (CR-39). Pour les traces de protons et de particules $\alpha$, la solution de gravure est l'hydroxyde de sodium $6,25 \mathrm{M}$ à $60^{\circ} \mathrm{C}$ avec des durées atteignant 21 heures. Pour les traces d'ions lithium (3 heures) et carbone (90 minutes), la gravure a été effectuée dans $\mathrm{NaOH} 7 \mathrm{M}$ à $70^{\circ} \mathrm{C}$ (d'après Vaginay, 2001 ; Fromm et al., 1991).

Etched track profiles and 3-D views for various ion tracks in CR-39. For the proton and alpha tracks, the etching conditions are $\mathrm{NaOH} 6.25 \mathrm{M}$ at $60^{\circ} \mathrm{C}$ with etching times up to 21 hours. For the Lithium (3 hours) and Carbon (90 minutes) tracks the etching conditions are $\mathrm{NaOH}$ 7M, $70{ }^{\circ} \mathrm{C}$ (Vaginay, 2001 ; Fromm et al., 1991).

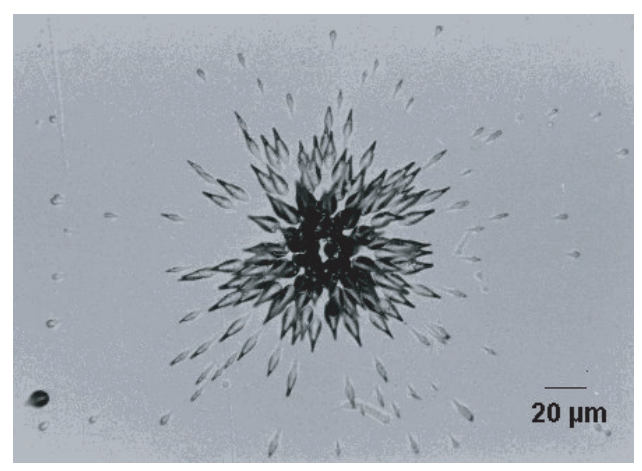

Figure 5 - Traces gravées dans le $\mathrm{CR}-39\left(\mathrm{NaOH} 6,25 \mathrm{M}, 60^{\circ} \mathrm{C}\right.$, 5 heures) après exposition de ce matériau à une particule chaude $\left({ }^{241} \mathrm{Am}\right)$ de dimensions micrométriques.

Etched tracks in CR-39 after irradiation with a hot particle of ${ }^{241}$ Am. 
représentant la vitesse spécifique $\mathrm{V}_{\mathrm{T}}$ pour un ion donné, il est possible non seulement de déterminer les paramètres géométriques d'une trace gravée pour un ion donné, mais aussi de calculer les angles critiques au-delà desquels la trace latente ne sera pas gravée et donc les angles au-delà desquels on perdra le signal (Fromm et al., 1996). Outre la notion d'angle critique, nous avons aussi modélisé le concept de temps différé, temps au bout duquel une trace gravée apparaît. En effet, dans certains cas, lorsqu'une trace est inclinée, la gravure ne permet pas de la rendre observable en raison de l'attaque globale du matériau qui ronge la surface du détecteur plus rapidement que la trace elle-même en raison de son inclinaison. Cependant, comme la vitesse $\mathrm{V}_{\mathrm{T}}$ peut être croissante, la trace gravée peut apparaître après un certain temps : le temps différé (Meyer et al., 1997).

\subsection{Mesure d'activité radon par DSTN}

La réponse de deux DSTN, le LR-115 (nitrate de cellulose) et le CR-39, a été déterminée expérimentalement sur la gamme des énergies des particules $\alpha$ émises par le radon et ses descendants, en utilisant un modèle de développement chimique des traces latentes (Barillon, 1994). Ces résultats, intégrés dans un modèle mathématique, ont permis de déterminer l'efficacité de détection d'un DSTN placé dans une cellule de mesure cylindrique pour un radioélément émetteur alpha donné. Confinée dans une membrane en polyéthylène permettant une bonne perméation du radon, la cellule, imperméable à l'eau et au thoron (isotope du radon contribuant au bruit de fond), peut être utilisée pour cartographier les activités en radon dans l'atmosphère, dans les sols et dans l'eau.

\subsection{La dosimétrie des neutrons par CR-39}

Les travaux sur les modèles de gravure chimique des traces ont conduit à l'évaluation du CR-39 en tant que dosimètre des rayonnements neutroniques.

Une modélisation complète a permis de simuler la conversion neutron-proton (n,p) au sein du CR-39 par la méthode de Monte Carlo, permettant la comptabilisation des événements engendrant des traces latentes au sein du DSTN, puis de calculer avec le modèle le nombre et l'ouverture de celles de ces traces qui sont gravées (Meyer, 1996).

La lecture des films de CR-39 se faisant sous microscope optique, celle-ci est de fait limitée par la densité des traces : au-delà d'une certaine densité, il n'est plus possible de séparer les traces et donc d'effectuer un comptage correct. Une autre voie a été proposée dans laquelle l'échantillon de CR-39 est éclairé par un faisceau laser He-Ne sous incidence normale, le flux de lumière diffractée par les traces révélées étant recueilli par une photodiode. Une simulation numérique, basée sur 
un modèle physique de diffraction de la lumière par les traces, a été réalisée, montrant une très bonne corrélation avec les mesures. Des mesures de diffusion de lumière appliquées à des CR-39 irradiés en présence d'un convertisseur hydrogéné par un spectre réaliste de neutrons ont été réalisées (Groetz et al., 1998 ; Groetz, 1997). Une équipe américaine du Georgia Institute of Technology et du Los Alamos National Laboratory a étendu ces travaux pour des lectures de dose neutronique comprise entre 0 et $50 \mathrm{mSv}$ (Moore et al., 2002).

\section{Le radon et ses mécanismes de diffusion}

Le radon-222 $\left({ }^{222} \mathrm{Rn}\right)$, gaz radioactif naturel produit par la désintégration du radium-226, lui-même descendant de l'uranium-238, est le principal composant de la radioactivité d'origine atmosphérique. Son étude est primordiale et le suivi de sa concentration fondamental dans le domaine des sciences de la terre, en tant que traceur, et dans le domaine de la radioprotection (installations minières, habitations et lieux publics, matériaux de construction).

\subsection{La métrologie du radon et de ses descendants}

Dans le cadre de la thèse de Klein (1990), un compteur proportionnel à flux gazeux a été réalisé pour la mesure en continu du ${ }^{222} \mathrm{Rn}$ et son application à des mesures de terrain. Susceptible de fonctionner sur des terrains hostiles comme les volcans, une cellule pour des traitements chimiques a été conçue, en utilisant un résonateur bimodal de cristal de quartz pour la mesure du dioxyde de soufre. Les différentes études de laboratoire ont permis de définir et de concevoir un prototype de terrain autonome, portable et modulaire.

D'autres voies ont été explorées, notamment la mesure des particules $\alpha$ par l'intermédiaire de photodiodes PIN de silicium. Ceci a fait l'objet de deux thèses (Devillard, 2000 ; Voytchev, 2000), dans lesquelles ont été développées des cellules de mesure aboutissant à la réalisation d'un compteur portable basé sur la détection des $\alpha$ émis par le ${ }^{222} \mathrm{Rn}(5,5 \mathrm{MeV})$, le ${ }^{218} \mathrm{Po}(6 \mathrm{MeV})$ et le ${ }^{214} \mathrm{Po}$ $(7,7 \mathrm{MeV})$. Ce compteur a été utilisé avec succès pour mesurer des concentration et débit de fluence en radon dans des anciennes mines et des habitations en France et en Bulgarie.

\subsection{Les mécanismes de transport et le rôle de l'eau}

En amont de la mesure, il est primordial de comprendre les mécanismes physiques gouvernant le transport du radon. En milieu sec ou humide, ces mécanismes sont définis par la désintégration du radium, la mécanique des fluides et l'écoulement 
en milieu poreux. Ainsi, deux processus sont considérés aux échelles microscopique et macroscopique :

- l'émanation, soit le passage du radon de la matrice cristalline vers l'espace poreux ;

- l'exhalation, qui est le transport du radon de l'espace poreux vers l'atmosphère.

Des travaux (Özgümüs, 2000) ont permis de comprendre dans quelles conditions il était possible de prévoir l'émanation du radon à partir de grandeurs expérimentalement mesurables sur des phases minérales. La schématisation du matériau poreux a conduit à l'élaboration de deux modèles complémentaires. Le premier représente le pore par l'espace entre deux matrices cristallines semiinfinies planes et parallèles. Quant au second modèle, il décrit le matériau comme un édifice construit par empilements de particules monodispersées, sphériques, pleines et lisses. Les résultats issus de ces modèles offrent de bonnes corrélations avec des expériences réalisées sur des fractions de résidus miniers uranifères, notamment en fonction de la teneur en eau. Ces modèles peuvent être directement appliqués à des échantillons dont certaines caractéristiques sont connues, telles que la distribution de la taille des pores, la porosité relative, la distribution des tailles de particules et la teneur en eau.

À l'échelle du matériau, le laboratoire a développé un modèle numérique, basé sur la méthode des éléments finis, simulant l'ensemble des processus entraînant l'exhalaison du radon de différents matériaux de construction (béton, ciment, plâtre, phosphogypse, etc.) dans l'atmosphère. Le calibrage du code de calcul a été effectué à partir de données expérimentales de concentration en radon obtenues par spectroscopie alpha sur des échantillons de phosphogypse, en collaboration avec l'université de Salzbourg (Autriche). L'étude numérique a été étendue aux autres matériaux pour mettre en évidence l'impact de la présence d'eau sur l'émanation et sur le transport du radon par diffusion. Il s'avère que l'eau engendre des modifications totalement différentes de la concentration et du taux d'exhalaison en radon, selon la porosité du matériau (Fig. 6). L'ensemble des résultats obtenus par simulation a offert de très bonnes corrélations avec les expériences réalisées en laboratoire sur des matériaux de construction de caractéristiques similaires (Fournier et al., 2005a, 2005b).

Cependant, les phénomènes à une échelle supérieure ne peuvent être définis qu'à travers des caractéristiques macroscopiques comme le potentiel d'exhalation des sols. Différentes propriétés géologiques et pédologiques peuvent alors intervenir, mais le principal paramètre gouvernant la variation du flux radon exhalé est la perméabilité, liée à l'état hydrique du sol (Demongeot, 1997). 


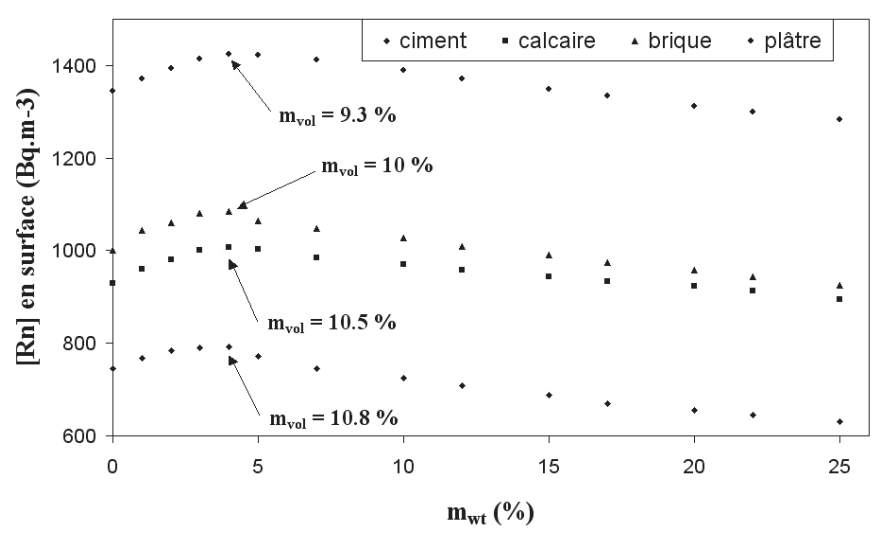

Figure 6 - Simulation de la concentration en radon à la surface de plusieurs matériaux en fonction de la teneur massique en eau.

Numerical simulation of the radon concentration at the interface between various samples according to the specific water content.

\section{Spectrométrie des rayonnements ionisants et radiobiologie}

Dans cette dernière partie, nous présentons les thèmes de recherche d'actualité au LMN-AC dans le domaine de la détection des rayonnements et de la radiobiologie.

\subsection{Spectrométrie des neutrons}

La spectrométrie et la dosimétrie des neutrons ont un domaine d'application large : radioprotection autour des accélérateurs et réacteurs (accélérateurs médicaux en radiothérapie, installations nucléaires de base), contrôle de matières inertes, caractérisation de matières fissiles, diagnostic plasma. Deux contraintes majeures font toujours l'objet d'intenses recherches :

- les spectres neutroniques, notamment auprès des installations nucléaires, s'étendent du domaine thermique aux neutrons rapides, représentant ainsi jusqu'à 10 ordres de grandeur ;

- les champs neutroniques impliquent toujours un rayonnement gamma, provenant soit des réactions induites par les neutrons (telles les sources PuBe ou $\mathrm{AmBe}$ ), soit des produits de fission, soit de l'activation de matériaux inertes : ce sont les champs mixtes $(\gamma, \mathrm{n})$.

Ces deux contraintes font qu'il est difficile d'avoir d'une part un appareil couvrant tout le spectre énergétique, ce qui induit toute une gamme de méthodes de 

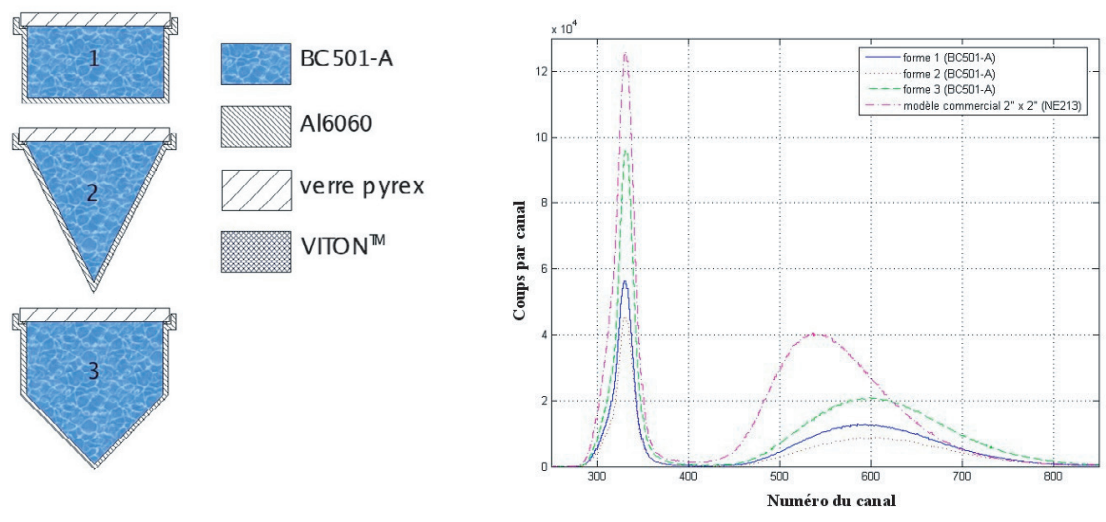

Figure 7 - Schéma des 3 formes sélectionnées en fonction de leur rapport efficacité neutron sur efficacité gamma. Figures de discrimination d'une forme commerciale et des 3 formes sélectionnées. Selected shapes built after simulation of neutron and gamma efficiencies. Pulse shape discrimination for a commercial cell and for the 3 selected shapes.

détection selon l'énergie incidente des neutrons (compteurs gazeux, chambres à fission, scintillateurs, détecteurs solides de traces nucléaires, dosimètres à bulles, dosimètres thermoluminescents, pastilles à activation, diodes, etc.) et d'autre part la perturbation due aux photons $\gamma$ nécessite de s'en affranchir autant que possible.

La caractérisation complète d'un ensemble de six compteurs gazeux à protons de recul est à l'étude, couvrant une échelle énergétique très large (de $0,025 \mathrm{eV}$ à $4,5 \mathrm{MeV}$ ). Les matrices de réponses des détecteurs sont reconstruites en tenant compte des phénomènes physiques inhérents à la détection (effets de paroi, inhomogénéité du champ électrique) afin d'obtenir une meilleure déconvolution des spectres de protons de recul (Benmosbah et al., 2006).

Une seconde étude a pour trait l'optimisation géométrique d'un scintillateur liquide. Ce type de détecteur est souvent utilisé pour l'acquisition de spectres neutron en champ mixte $\mathrm{n} / \gamma$, car très rapide et possédant un grand pouvoir de discrimination entre les neutrons et les photons $\gamma$. Cependant, son domaine de détection ne descend guère en dessous de $1 \mathrm{MeV}$ et la discrimination $\mathrm{n} / \gamma$ est d'autant moins efficace que les photons $\gamma$ sont majoritaires. Nous avons donc cherché à optimiser les paramètres géométriques d'un scintillateur liquide organique de type BC501A, en tenant compte des efficacités dues aux photons $\gamma$ et aux neutrons. Trois nouvelles formes ont ainsi été sélectionnées (Fig. 7) et leurs figures de discrimination neutron/ $\gamma$ ont été nettement améliorées par rapport aux formes commerciales (Begin et al., 2006). Leurs fonctions de réponses sont 

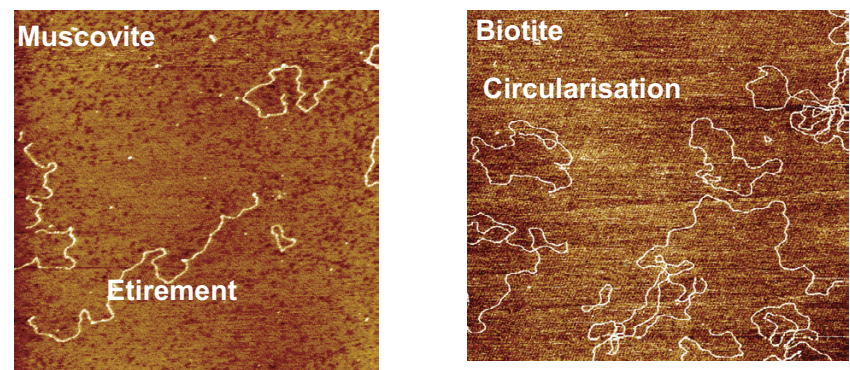

Figure 8-Observation par AFM de fragments d'ADN du phage lambda sur la muscovite $\left(3 \times 3 \mu^{2}\right.$, $z=3 \mathrm{~nm})$ et sur la biotite $\left(3 \times 3 \mu^{2}, z=3 \mathrm{~nm}\right)$ : mise en évidence de l'influence de la nature du substrat.

AFM observation of DNA fragments (lambda phage) deposited on muscovite $\left(3 \times 3 \mu^{2}\right.$, $z=3 \mathrm{~nm})$ and biotite $\left(3 \times 3 \mathrm{\mu m}^{2}, z=3 \mathrm{~nm}\right)$ micas : the influence of the type of mica (di- or trioctahedral) on the DNA conformation is clearly demonstrated.

déterminées par simulation Monte Carlo, afin de restituer avec la plus grande précision des spectres sur réacteur solide.

\subsection{Radiobiologie}

\subsubsection{Immobilisation de molécules d'ADN sur substrat solide}

La première étude en rapport avec la radiobiologie au LMN-AC fut un travail à caractère pluridisciplinaire ayant pour objectif d'étudier l'immobilisation de molécules d'ADN sur mica pour leur observation en microscopie à force atomique (AFM). Pour combler le manque de contrôle du protocole d'étalement des molécules sur mica, il a fallu isoler les différents paramètres intervenant durant le dépôt et mettre en évidence leur rôle sur l'immobilisation et l'étalement des molécules. Ces paramètres sont la concentration du chlorure de magnésium, la nature du substrat de mica et le type de la solution tampon (TRIS-HEPES, etc.). L'étude a été réalisée sur deux classes de micas ayant des propriétés différentes et donc réagissant différemment au traitement par la solution d'ADN. Les micas dioctaédriques interagissent principalement avec le tampon (TRIS) qui joue dans ce cas le rôle d'agent de fixation des molécules. Quant au magnésium, il interagit par création de complexes sur l'ADN et joue ainsi un rôle «protecteur». Les micas trioctaédriques interagissent peu avec le tampon mais sont susceptibles d'être altérés en volume par la présence de magnésium. Ces interactions vont induire des changements de conformation des molécules étalées en fonction des conditions (Fig. 8). Une validation immédiate de ces résultats a été obtenue en les appliquant à l'analyse par AFM des modifications induites par un rayonnement 


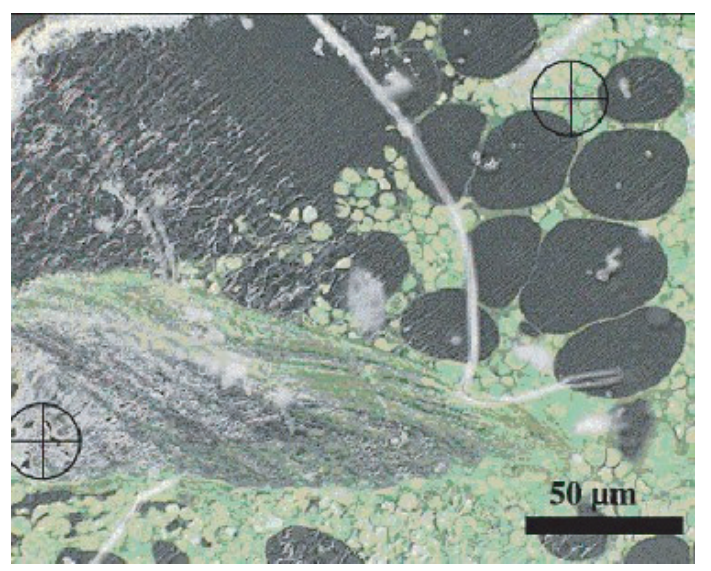

Figure 9 - Localisation de deux événements de fission de noyaux de neptunium présents dans une coupe de moelle osseuse.

Localisation of two fission fragments emission located in a bone marrow thin slice.

ionisant sur l'ADN. Nous avons en particulier étudié l'effet scavenger de la solution tris et l'effet de la concentration en ADN. Ces résultats, en parfait accord avec les données obtenues à l'aide de techniques classiques, ont confirmé l'intérêt de l'AFM dans le domaine de la radiobiologie (Boichot, 2000 ; Boichot et al., 2002).

\subsubsection{Rayonnements et cellules}

La distribution tissulaire et les biocinétiques d'élimination du neptunium-237 sont relativement bien connues, mais les données concernant sa distribution intracellulaire sont peu nombreuses, disparates et ne permettent pas d'expliquer la difficulté présentée quant à la décorporation de ce radionucléide. Nous avons cherché à déterminer les cibles cellulaires de concentration de ce radioélément artificiel de très longue période, tout en s'attachant à définir son influence sur le vivant.

Deux approches ont été envisagées afin de localiser le ${ }^{237} \mathrm{~Np}$ au niveau cellulaire. La première est une étude histologique reposant sur la mise en œuvre des microscopies électronique et ionique, ce qui a permis de confirmer une concentration intranucléaire dans le foie et le rein (Pusset et al., 2002). Puis une méthode basée sur la microcartographie des fragments de fission, issus de l'irradiation neutronique de coupes organiques contenant du ${ }^{237} \mathrm{~Np}$, a été développée (Fig. 9). Ce procédé consiste à induire la fission d'atomes de ${ }^{237} \mathrm{~Np}$ 
présents dans une coupe organique et à enregistrer la position de ces fissions avec un DSTN, couplé à un système de repérage micrométrique (Pusset et al., 2005).

À partir de ces méthodes, les effets toxiques du ${ }^{237} \mathrm{~Np}$ ont été démontrés en observant une densité importante de lésions apoptotiques au sein des tissus de foie, de reins et de moelle osseuse (Pusset et al., 2003). Une corrélation de la concentration de neptunium et de l'apparition de ces lésions a été effectuée et le problème de la nature de la toxicité du neptunium a été soulevé (Pusset, 2004).

D'autres travaux d'importance ont concerné la propagation de l'effet de proximité, c'est-à-dire la communication d'effets délétères entre des cellules irradiées par des particules $\alpha$ et leurs proches voisines. Ce phénomène, caractéristique aux faibles doses, s'apparente à une transmission de signaux biochimiques entre la faible proportion de cellules traversées par les particules et les cellules non irradiées dans une monocouche. Il résulte en une amplification de réponse biologique dont les mécanismes sous-jacents sont encore méconnus.

Il a fallu mettre au point un dispositif permettant de matérialiser le passage de chaque particule traversant la monocouche cellulaire, afin d'établir une microcartographie fidèle de l'irradiation puis de superposer celle-ci aux images présentant l'expression de la réponse cellulaire in situ. Pour cela, des enceintes de cultures intégrant un polymère DSTN d'épaisseur micrométrique dans leur support cellulaire ont été conçues, permettant la localisation précise de l'impact. Les enjeux technologiques soulevés ont nécessité une conception entièrement contrôlée des enceintes, combinant le moulage du détecteur sous forme de film micrométrique, la photo-polymérisation du DSTN et son greffage sous le support cellulaire par copolymérisation photo-amorcée. Leur utilisation a été pleinement validée pour établir la superposition désirée (Gaillard et al., 2005a).

Dans une seconde étape, les propriétés de détection des enceintes ont été mises à profit afin de qualifier un effet de proximité, caractérisé par la présence d'une surexpression intranucléaire de protéines $\mathrm{P} 21$ en réponse précoce à l'endommagement de l'ADN des cellules. La superposition traces/réponse a permis de définir les critères spécifiques de l'irradiation qui sont à l'origine de l'effet de proximité. Il est ainsi possible d'identifier et de co-localiser les cellules « émettrices de signaux » et les cellules « réceptrices de signaux » impliquées lors de la communication intercellulaire. En outre, les études menées ont permis de déterminer l'amplification de réponse suite à une exposition de très faible dose (Gaillard et al., 2005b). Enfin, les images superposées (traces/réponse) ont fait l'objet d'une série systématique d'analyses mathématiques dans le but d'accéder aux premiers résultats métrologiques de la transmission de signaux par effet de proximité. 


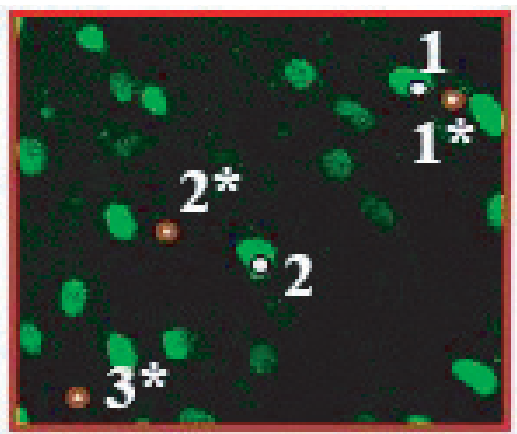

Figure 10 - Localisation d'impacts de particules $\alpha$ sur une monocouche de fibroplastes (AG1522) irradiée avec une dose moyenne de 0,13 cGy. Les numéros comportant un astérisque concernent des impacts hors des noyaux cellulaires. Ces derniers présentent une coloration d'autant plus forte que la protéine P21 (arrêt cellulaire) est surexprimée.

Localisation of alpha particles impacts in a fibroblasts (AG1522) layer irradiated with a mean dose of 0.13 cGy. The numbers with a star (*) indicate impacts out of the cell nucleus. These last compartments exhibit a colour intensity that is proportional to the P21 (cell-arrest) overexpression rate.

\section{Conclusion}

Les activités de recherche du Laboratoire de Microanalyses Nucléaires Alain Chambaudet ont historiquement débuté avec l'étude des rayonnements ionisants dans des matrices minérales ou des matériaux polymères. Sont venus s'additionner au cours du temps des compétences et travaux sur la spectrométrie des rayonnements, puis sur le vivant avec la radiobiologie. D'autres voies de recherche sont maintenant bien implantées au laboratoire, notamment les capteurs de gaz et les mécanismes moléculaires d'oxydation dans les milieux d'intérêt biologique.

\section{RÉFÉRENCES}

Barillon R. (1994) Contribution à la détection de particules alpha par détecteurs solides de traces nucléaires - Application à la mesure du radon, Thèse de l'université de Franche-Comté.

Begin F., Assaillit G., Groetz J.E. (2006) New shapes for liquid scintillation detectors used in neutron spectrometry, Nucl. Instrum. Meth. A 562, 351-357.

Benmosbah M., Groetz J.E., Crovisier P., Asselineau B., Van-Ryckeghem L., Truffert H., Cadiou A., Ziarnowski D. (2006) A comprehensive study of a wide energy range proportional counter neutron spectrometer, Proceedings of Science, http://pos.sissa.it//archive/conferences/025/005/ FNDA2006_005.pdf.

Boichot S. (2000) Immobilisation de molécules d'ADN sur mica pour leur étude en microscopie à force atomique. Application à l'analyse des modifications induites par un rayonnement ionisant, Thèse de l'université de Franche-Comté. 
Boichot S., Fromm M., Cunniffe S., O’Neill P., Labrune J.C., Chambaudet A., Delain E., Le Cam E. (2002) AFM investigation or radiation damage to DNA, Radiat. Prot. Dosim. 99, 143-145.

Cartwright B.G., Shirk E.K., Price P.B. (1978) A Nuclear-track-recording polymer of unique sensitivity and resolution, Nucl. Instrum. Meth. 153, 457-460.

Cassou R.M., Benton E.V. (1978) Properties and applications of CR-39 polymeric nuclear track detector, Nucl. Track Detection 2, 173-179.

Demongeot S. (1997) Recherche des différents paramètres caractérisant le potentiel d'exhalation en radon des sols, Thèse de l'université de Franche-Comté.

Devillard C. (2000) Caractérisation d'une photodiode silicium pin pour la détection des particules alpha - Application à la mesure de sources solides diffuses, Thèse de l'université de Franche-Comté.

Ditlov V.A., Awad E.M., Fromm M., Hermsdorf D. (2005) The Bragg-peak studies in CR-39 on the base of many-hit model for track etch rates, Radiat. Meas. 40, 249-254.

Fleischer R.L., Price P.B., Walker R.M. (1975) Nuclear Tracks in Solids: Principles and Applications, University of California Press, Berkeley.

Fournier F., Groetz J.E., Jacob F., Crolet J.M., Lettner H. (2005a) Simulation of radon transport through building materials : influence of the water content on radon exhalation rate, Transport in porous media 59, 197-214.

Fournier F., Groetz J.E., Jacob F., Lettner H., Crolet J.M., Chambaudet A. (2005b) Study of the Radon222 exhalation rate of phosphogypsum blocks: comparison with modelling. In: The natural radiation environment, seventh international symposium on the Natural Radiation Environment (Mc Laughlin J.P., Simopoulos S.E., Steinhaüsler F., Eds), Rhodes, 20-24 May 2002, pp. 582589.

Fromm M., Membrey F., Chambaudet A., Saouli R. (1991) Proton and alpha track profiles in CR39 during etching and their implications on track etching models, Nucl. Tracks Radiat. Meas. 19. 163-168.

Fromm M., Membrey F., Chambaudet A, Saouli R., Al-Rahamany A. (1993) A study of CR39 bulk etch properties under various temperature and concentration conditions to modelize the dissolution rate, Nucl. Tracks Radiat. Meas. 21, 357-365.

Fromm M., Meyer P., Chambaudet A. (1996) Ion track etching in isotropic polymers: etched track shape and detection efficiency, Nucl. Instrum. Meth. B 107, 337-343.

Fromm M. (2005) Light MeV-ions etching studies in a plastic track detector, Radiat. Meas. 40, 160-169.

Gaillard S., Armbruster V., Hill M.A., Gharbi T., Fromm M. (2005a) Studies of UV-cured CR-39 recording properties in view of its applicability in radiobiological experiments with alpha particles, Radiat. Meas. 40, 279-282.

Gaillard S., Armbruster V., Hill M.A., Gharbi T., Fromm M. (2005b) Production and validation of CR39 based dishes for $\alpha$-particle radiobiological experiments, Radiat. Res. 163, 343-350.

Groetz J.E. (1997) Diffusion de lumière cohérente par des traces nucléaires dans le CR-39 ; Application à la dosimétrie des rayonnements, Thèse de l'université de Franche-Comté.

Groetz J.E., Lacourt A., Chambaudet A. (1998) Coherent light scattering by nuclear etched tracks in the PADC (form of CR-39), Nucl. Instrum. Meth. B 142, 503-514.

Klein D. (1990) Réalisation et applications d'un compteur proportionnel pour des mesures de terrain en continu du radon 222, Thèse de l'université de Franche-Comté.

Meesen, G., Poffijn, A. (2001) Semi-automated analysis of three-dimensional track images, Radiat. Meas. 34, 161-165.

Meyer P. (1996) Propriétés du CR-39 en dosimétrie neutron « faible flux » : Étude de la conversion par une méthode de Monte Carlo et simulation numérique de la gravure chimique des traces latentes, Thèse de l'université de Franche-Comté. 
Meyer P., Jouffroy M., Membrey F., Fromm M., Chambaudet A. (1997) Parameters influencing the existence and the observability of etched tracks: Application to alpha particle detection in CR39, Radiat. Prot. Dosim. 74, 75-83.

Moore M.E., Gepford H.J., Hernes R.E., Hertel N.E., Devine R.T. (2002) Laser illuminated etched track scattering (LITES) dosimetry system, Radiat. Prot. Dosim. 101, $43-45$.

Özgümüs A. (2000) Contribution à la modélisation de l'émanation du radon par effet de recul direct. Influence de l'eau, Thèse de l'université de Franche-Comté.

Pusset D. (2004) Approches microscopiques et développement d'une méthode de microcartographie pour la localisation du neptunium-237 à l'échelle cellulaire. Mise en évidence de processus apoptotiques in vivo, Thèse de l'université de Franche-Comté.

Pusset D., Fromm M., Poncy J.L., Kantelip B., Galle P., Chambaudet A., Baud M., Boulahdour H. (2002) Ultrastructural lesions induced by neptunium-237: apoptosis or nécrosis? Can. J. Physiol. Pharmacol. 80, 654-661.

Pusset D., Boulahdour H., Fromm M., Poncy J.L., Kantelip B., Griffond B., Baud M., Galle P. (2003) Ultrastructural apoptotic lesions induced in bone marrow after neptunium-237 contamination, Anticancer Res. 23, 4837-4842.

Pusset D., Fromm M., Boulahdour H., Marsault D., Gharbi T., Tsuruta T., Kantelip B., Poncy J.L. (2005) Cellular localisation of neptunium-237 in biological tissue by fission fragments cartography, Radiat. Meas. 40, 289-294.

Vaginay, F. (2001) Analyse de traces nucléaires 3-D par microscopie confocale : Détermination des fonctions de réponse, Thèse de l'université de Franche-Comté.

Voytchev M. (2000) Étude et développement d'une métrologie des particules alpha utilisant la technologie des photodiodes - Application à la mesure du radon et de ses descendants, Thèse de l'université de Franche-Comté.

Young D.A. (1958) Etching of radiation damage in lithium fluoride, Nature 182, 375-377. 